\title{
Case Report \\ Photosensitive Epilepsy and Polycystic Ovary Syndrome as Manifestations of MERRF
}

\author{
Josef Finsterer (ii) \\ Klinik Landstrasse, Vienna, Austria \\ Correspondence should be addressed to Josef Finsterer; fipaps@yahoo.de
}

Received 8 July 2020; Revised 8 September 2020; Accepted 18 September 2020; Published 29 September 2020

Academic Editor: Peter Berlit

Copyright (c) 2020 Josef Finsterer. This is an open access article distributed under the Creative Commons Attribution License, which permits unrestricted use, distribution, and reproduction in any medium, provided the original work is properly cited.

Objectives. Although endocrinologic involvement and epilepsy are frequent features of myoclonic epilepsy with ragged-red fibers (MERRF), polycystic ovary syndrome (PCOS) and photosensitive epilepsy have not been reported. Case Report. A 32-year-old female was diagnosed with MERRF at age $19 \mathrm{y}$ upon presence of the four canonical features and the variant $\mathrm{m} .8344 \mathrm{~A}>\mathrm{G}$ in $M T$ TK (tRNA (Lys)) (blood heteroplasmy rate: 50\%). She experienced recurrent photosensitive focal and generalised seizures since age $19 \mathrm{y}$, which could be triggered by flickering light or by looking at small stones, leaves, or dirty snow on the ground. Since the last 42 months, she was seizure-free upon levetiracetam $(4000 \mathrm{mg} / \mathrm{d})$, clonazepam $(1.5 \mathrm{mg} / \mathrm{d})$, and topiramate $(25 \mathrm{mg} / \mathrm{d})$. Additionally, she suffered from secondary amenorrhoea since adolescence. She was married between ages $19 \mathrm{y}$ and $25 \mathrm{y}$ but did not get pregnant. PCOS was diagnosed and treated with desogestrel plus estradiol. Nonetheless, the course was progressive, particularly with regard to ataxia, myocloni, and myopathy. Conclusions. The phenotypic spectrum of MERRF is broader than anticipated and may additionally include PCOS and photosensitive epilepsy. PCOS in MERRF may respond to hormone substitution and photosensitive epilepsy to levetiracetam, clonazepam, and topiramate.

\section{Introduction}

Myoclonic epilepsy with ragged-red fibers (MERRF) is a rare syndromic mitochondrial disorder (MID) due to currently 26 mutations in 15 different genes [1]. The most frequent of these mutations is the variant $\mathrm{m} .8344 \mathrm{~A}>\mathrm{G}$ in $M T-T K$ (tRNA (Lys)) [1]. Although MERRF is clinically characterised by the four canonical features, ataxia, epilepsy, myoclonus, and myopathy [1-3], it is a multisystem disorder with a much broader phenotypic spectrum (MERRF-plus) [1,2]. Organs affected in addition to the brain and the muscle in MERRF-plus include the peripheral nerves, eyes, ears, heart, gastrointestinal tract, or the endocrine organs [1]. Although involvement of the brain and endocrine system in MERRF-plus is well appreciated $[3,4]$, polycystic ovary syndrome (PCOS) and photosensitive epilepsy have not been reported as a phenotypic manifestation of MERRF-plus.

\section{Case Report}

The patient is a 32-year-old Caucasian female, of height $166 \mathrm{~cm}$ and weight $50 \mathrm{~kg}$, with uneventful early development who became noteworthy at age $7 \mathrm{y}$ because of poor school performance due to impaired memory and concentration. She had nocturnal enuresis until age $14 \mathrm{y}$ and menarche at age $13 \mathrm{y}$ with normal periods thereafter. At age $17 \mathrm{y}$, recurrent, spontaneous myocloni of all four limbs began. A first generalised tonic-clonic seizure occurred at age $19 \mathrm{y}$. The postictal period was characterised by severe muscle weakness and muscle aching during $24 \mathrm{~h}$. Electroencephalography (EEG) recording revealed epileptiform discharges in the frontocentral regions under hyperventilation and a decreased photoparoxysmal threshold. Valproic acid (VPA) was begun.

Despite VPA, seizures recurred with a frequency of 1-2 seizures/month. In addition to generalised seizures, she 
4.5

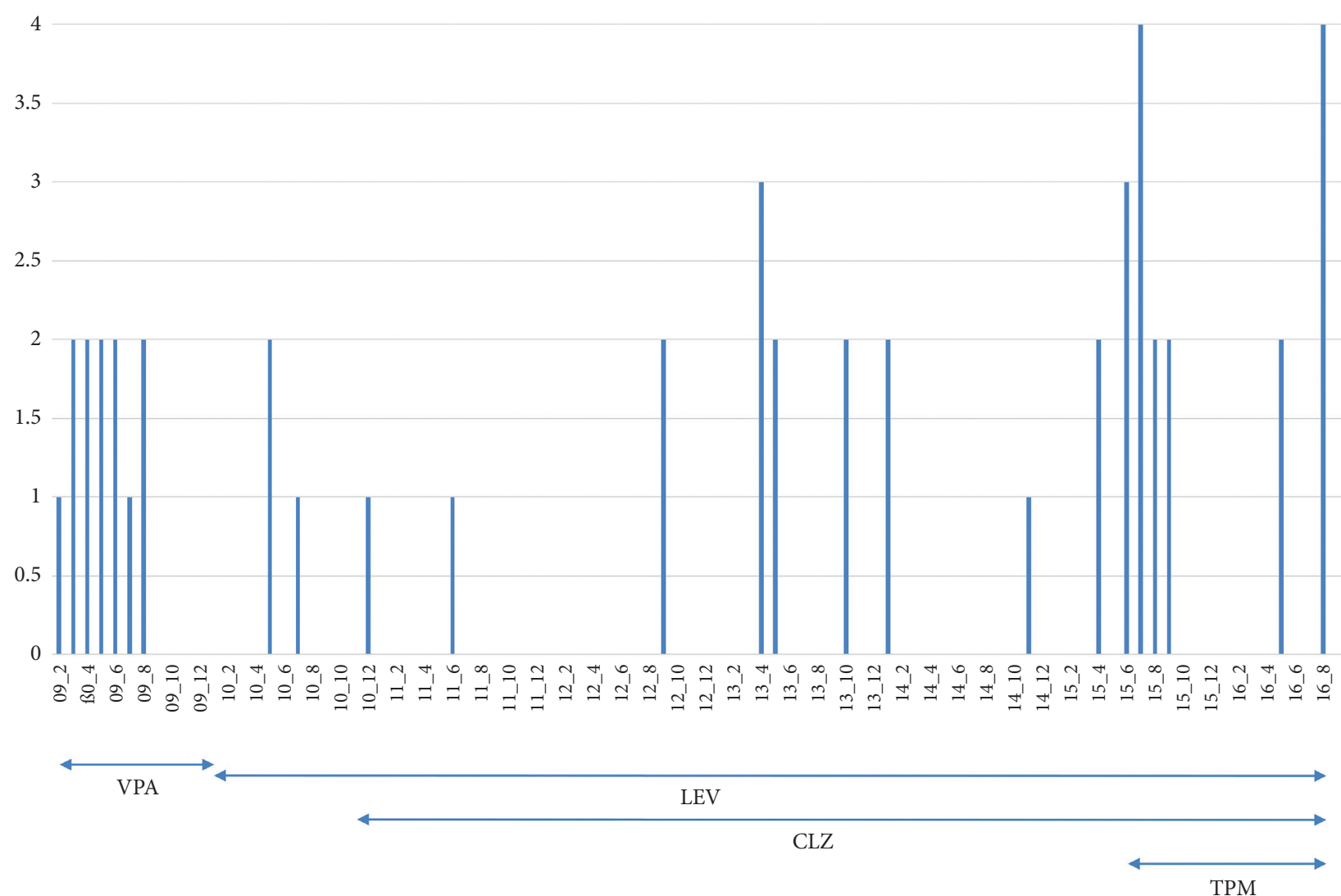

FIgURe 1: Frequency of focal and generalised seizures since onset at age $19 \mathrm{y}$ in the index patient (1): focal seizure, (2): one generalised seizure, (3): two generalised seizures, (4): three generalised seizures per day).

rarely experienced focal seizures (Figure 1). Seizures could be triggered by flickering light or by looking at small stones, leaves, or dirty snow on the ground. Walking safely for $1 \mathrm{~km}$ with one stop was possible only after sunset. Nerve conduction studies revealed a mixed axonal/demyelinating neuropathy. Electromyography was myogenic. MRI of the brain at ages $19 \mathrm{y}, 21 \mathrm{y}$, and $28 \mathrm{y}$ revealed cerebellar atrophy exclusively. At age $20 \mathrm{y}$, VPA was replaced by levetiracetam (LEV) $3000 \mathrm{mg} / \mathrm{d}$. Genetic work-up at age 21 y revealed the variant $\mathrm{m} .8344 \mathrm{~A}>\mathrm{G}$ with a heteroplasmy rate of $50 \%$ in blood lymphocytes. EEG at age $24 \mathrm{y}$ revealed bilaterally synchronous spike-wave complexes in the occipital area. Flickering light triggered generalised, bilaterally synchronous spike-wave and polyspike-wave complexes (Figure 2). The patient had been married between ages $19 \mathrm{y}$ and $25 \mathrm{y}$ but never became pregnant. Work-up for infertility at age $20 \mathrm{y}$ revealed a PCOS for which she received desogestrel $(150 \mu \mathrm{g} /$ d) + estradiol $(20 \mu \mathrm{g} / \mathrm{d})$ twice a year for three months since age $26 y$. Since age $27 y$, ataxic gait worsened such that she required support from another person for walking. Elevated serum lactate levels up to double the upper reference limits were noted for the first time. At age $27 \mathrm{y}$, topiramate (TPM) was added. Seizures had not recurred during 42 months prior to the last follow-up at age 32 y (Figure 1).

The family history was positive for easy fatigability (mother (48yo), brother (26yo), great aunt from mother's side), photosensitivity (mother, grandmother from mother's side, 2 great aunts from mother's side, 2 aunts and 1 uncle from mother's side), hand tremor (brother), myocloni (grandmother from mother's side, 2 aunts, 1 uncle, 2 cousins from the mother's side), seizures (1 great aunt from the mother's side, 1 cousin), Parkinsonism (1 great aunt from the mother's side, 1 uncle), ataxia (2 great aunts from the mother's side), and hypothyroidism (mother) (Figure 3). The mother felt unpleasant with flickering light and when looking at many stones, leaves, or dirty snow on the ground. Both mother and brother of the index patient carried the m.8344A > G variant, the mother had a heteroplasmy rate of $40 \%$ and the brother was homoplasmic. Clinical neurologic exam of the index patient at age $32 \mathrm{y}$ revealed cognitive impairment, ataxia, photosensitivity, dysarthria, uncoordinated speech, myocloni of upper limbs, generalised wasting, absent tendon reflexes, and gait disturbance. The current medication included LEV $(4000 \mathrm{mg} / \mathrm{d})$, clonazepam (CLZ) 


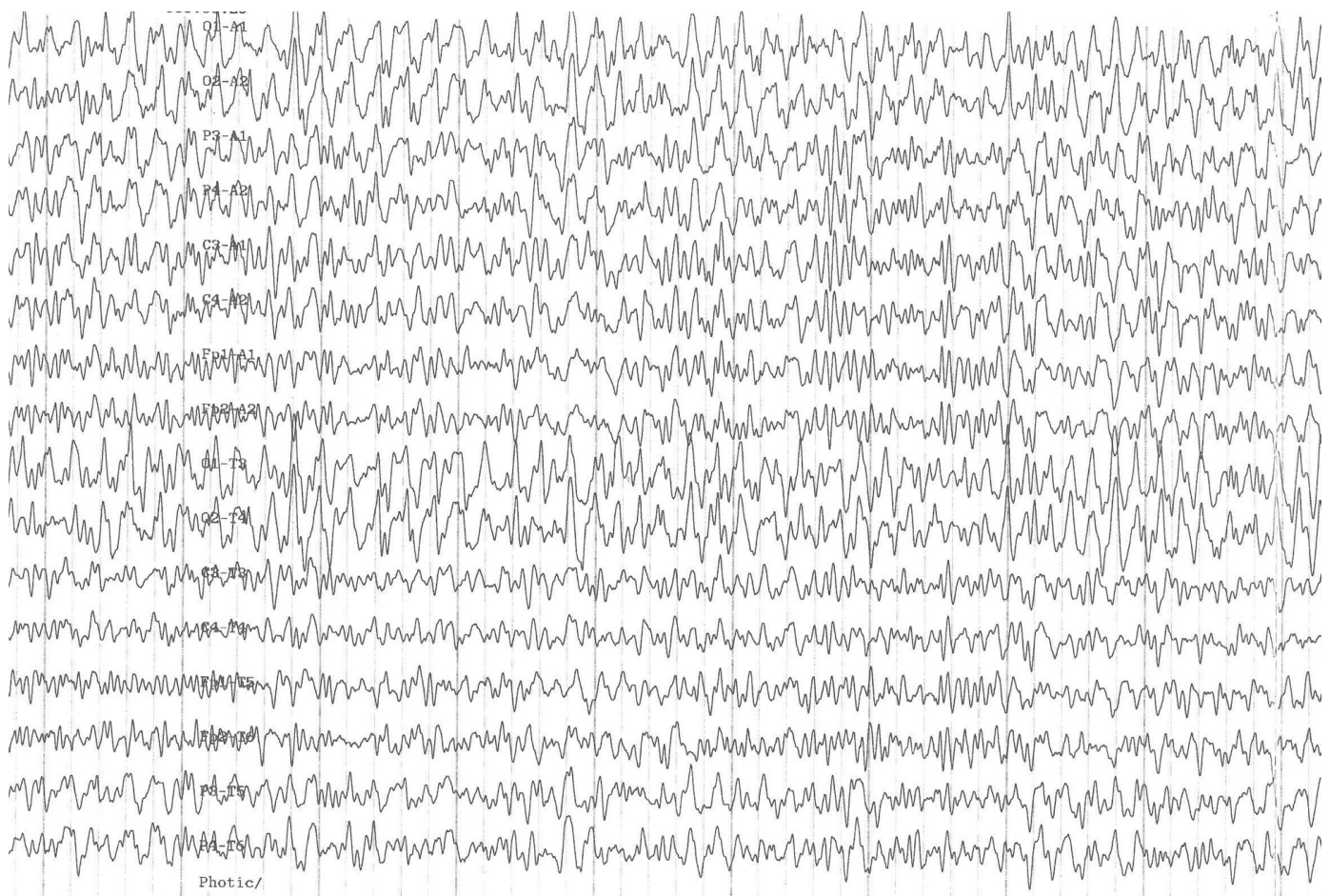

FIGURE 2: EEG under flickering light at age 24 y showing generalised, bilaterally synchronous spike-wave and polyspike-wave complexes.

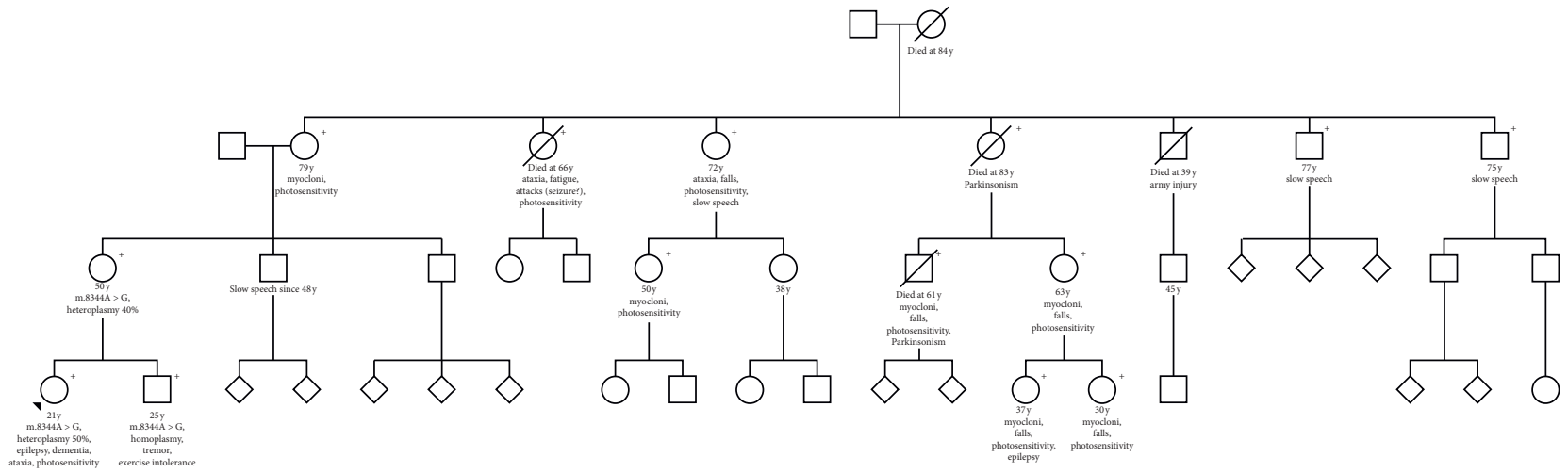

FIgURE 3: Pedigree of the index patient's family (the “+” indicates that the individual was clinically affected).

$(1.5 \mathrm{mg} / \mathrm{d}), \quad$ TPM $\quad(25 \mathrm{mg} / \mathrm{d})$, coenzyme-Q $\quad(200 \mathrm{mg} / \mathrm{d})$, L-carnitine $(2 \mathrm{~g} / \mathrm{d})$, and vitamin-E $(400 \mathrm{mg} / \mathrm{d})$.

\section{Discussion}

The presented patient is interesting for the following aspects. First, the patient had MERRF, which is still a rare MID syndrome. MERRF was diagnosed upon presence of all four canonical features and the m.8344A $>\mathrm{G}$ variant. The index patient had inherited the disease from her mother who was only mildly affected. The low heteroplasmy rate of $50 \%$ does not exclude pathogenicity, as it had been determined only in blood.

Second, the patient had photosensitive epilepsy, which has not been reported in MERRF. It is characterised by the occurrence of seizures after exposure to certain visual stimuli.
Many patients experience an aura prior to onset of the seizure or feel odd sensations before the seizure occurs [5]. Interestingly, the same triggers that induced epilepsy in the index patient induced only an unpleasant feeling in the mother. Although the mother had never recorded an EEG, it is conceivable that the unpleasant feeling actually represents focal seizures.

Third, the index patient had PCOS, which has not been reported in MERRF. PCOS was speculated to have been triggered by VPA, as has been previously reported [6], but the question if VPA truly causes PCOS is still under debate $[7,8]$. A strong argument against VPA as the trigger of PCOS is that PCOS has been reported in MIDs other than MERRF and not under VPA $[9,10]$. Thus, it is more likely that PCOS in the index patient was a manifestation of the underlying MID than a side effect of VPA. A further argument against VPA is that many MID patients received VPA without 
developing PCOS. Whether VPA enhanced the preexisting endocrine problem remains speculative.

Fourth, homoplasmy of the m.8344A $>\mathrm{G}$ variant has been only rarely reported $[11,12]$. Whether patients carrying a homoplasmic m.8344A > G variant are more severely affected than heteroplasmic patients is unknown but the few cases available suggest that onset is earlier and the phenotype is more severe in homoplasmic than heteroplasmic patients.

Limitations of the study are that no muscle biopsy had been taken, that heteroplasmy was determined only in blood lymphocytes, that no prospective investigations for multisystem involvement had been carried out, that firstdegree relatives were not systematically investigated, and that the progression of the disease had been only faultily monitored.

Overall, this case shows that the phenotypic spectrum of MERRF-plus is broader than anticipated and may additionally include PCOS and photosensitive epilepsy. PCOS in MERRF may respond to hormone substitution and photosensitive epilepsy to LEV, CLZ, and TPM.

\section{Conflicts of Interest}

The author declares that there are no conflicts of interest.

\section{Authors' Contributions}

J. F. is responsible for design, literature search, discussion, writing of the first draft, and final approval.

\section{References}

[1] J. Finsterer, S. Zarrouk-Mahjoub, and J. M. Shoffner, "Merrf classification: implications for diagnosis and clinical trials," Pediatric Neurology, vol. 80, pp. 8-23, 2018.

[2] S. DiMauro and M. Hirano, GeneReviews ${ }^{\circledR}$, University of Washington, Seattle, WA, USA, 2003, http://www.ncbi.nlm. nih.gov/books/NBK1520/.

[3] J. Finsterer, "Pharmacotherapeutic management of epilepsy in merrf syndrome," Expert Opinion on Pharmacotherapy, vol. 20, no. 10, pp. 1289-1297, 2019.

[4] N. Ohkoshi, A. Ishii, N. Shiraiwa, S. Shoji, and K. Yoshizawa, "Dysfunction of the hypothalamic-pituitary system in mitochondrial encephalomyopathies," Journal of Medicine, vol. 29, pp. 13-29, 1998.

[5] A. Martins Da Silva and B. Leal, "Photosensitivity and epilepsy: current concepts and perspectives-a narrative review," Seizure, vol. 50, pp. 209-218, 2017.

[6] Y. Kawakami, S. Fujii, G. Ishikawa, A. Sekiguchi, A. Nakai, and M. Takase, "Valproate-induced polycystic ovary syndrome in a girl with epilepsy: a case study," Journal of Nippon Medical School, vol. 85, no. 5, pp. 287-290, 2018.

[7] H. Goldberg-Stern, E. Yaacobi, M. Phillip, and L. De Vries, "Endocrine effects of valproic acid therapy in girls with epilepsy: a prospective study," European Journal of Paediatric Neurology, vol. 18, no. 6, pp. 759-765, 2014.

[8] D. Gotlib, R. Ramaswamy, J. E. Kurlander, A. DeRiggi, and M. Riba, "Valproic acid in women and girls of childbearing age," Current Psychiatry Reports, vol. 19, no. 9, p. 58, 2017.

[9] Y. Ding, B.-H. Xia, C.-J. Zhang, and G.-C. Zhuo, "Mitochondrial tRNALeu (UUR) C3275T, tRNAGln T4363C and
tRNALys A8343G mutations may be associated with PCOS and metabolic syndrome," Gene, vol. 642, pp. 299-306, 2018.

[10] J. Finsterer and S. Zarrouk-Mahjoub, "Polycystic ovary syndrome in mitochondrial disorders due mtDNA or nDNA variants," American Journal of Translational Research, vol. 10, no. 1, pp. 13-15, 2018.

[11] P. Buda, D. Piekutowska-Abramczuk, A. KarkucińskaWięckowska et al., "Drop attacks as first clinical symptoms in a child carrying MTTK m.8344A > G mutation," Folia Neuropathologica, vol. 4, pp. 347-354, 2013.

[12] Y. Monden, M. Mori, M. Kuwajima, T. Goto, T. Yamagata, and M. Y. Momoi, "Late-onset leigh syndrome with myoclonic epilepsy with ragged-red fibers," Brain and Development, vol. 35, no. 6, pp. 582-585, 2013. 